\title{
On the Rate of Convergence to Equilibrium in One-Dimensional Systems ${ }^{\star}$
}

\section{Gerhard Keller}

Institut für Angewandte Mathematik, Im Neuenheimer Feld 294, D-6900 Heidelberg, Federal Republic of Germany

\begin{abstract}
We determine the essential spectral radius of the Perron-Frobeniusoperator for piecewise expanding transformations considered as an operator on the space of functions of bounded variation and relate the speed of convergence to equilibrium in such one-dimensional systems to the greatest eigenvalues of generalized Perron-Frobenius-operators of the transformations (operators which yield singular invariant measures).
\end{abstract}

\section{Introduction}

In this note we give some estimates on the speed of convergence to equilibrium in 1-dimensional dynamical systems which can be described by a piecewise monotonic transformation $T:[0,1] \rightarrow[0,1]$. "Piecewise monotonic" means that there is a finite partition $\mathscr{I}$ of $[0,1]$ into intervals on each of which $T$ is strictly monotone and differentiable. Throughout the paper we assume the following setting (see $[4,13])$ :

$g:[0,1] \rightarrow \mathbb{R}_{+}$is defined by

$$
g(x)=1 /\left|T^{\prime}(x)\right| \text { for } x \in X_{0}:=\bigcup_{I \in \mathscr{I}} \operatorname{int} I,
$$

$g(x) \leqq \liminf _{y \rightarrow x, y \in X_{0}} g(y)$ on the finite set $[0,1] \backslash X_{0}$. Set

$$
g_{n}(x)=g\left(T^{n-1} x\right) \cdot \ldots \cdot g(x)
$$

and $\vartheta=\lim _{n \rightarrow \infty}\left(\left\|g_{n}\right\|_{\infty}\right)^{1 / n}, P: L^{1} \rightarrow L^{1}$ is defined by

$$
P f(x)=\sum_{I \in \mathscr{I}}(f \cdot g)\left(T_{\mid I}^{-1}(x)\right) \cdot 1_{T I}(x) .
$$

( $L^{1}$ is the space of complex-valued Lebesgue-integrable functions on $[0,1] ; P$ is the Perron-Frobenius-operator associated

* This work has been supported by the Deutsche Forschungsgemeinschaft 
to $T$ and does not depend on the choice of $g(x)$ for $x \notin X_{0}$.) $P$ satisfies

$$
\int P f_{1}(x) \cdot f_{2}(x) d x=\int f_{1}(x) \cdot f_{2}(T x) d x
$$

for $f_{1} \in L^{p}, f_{2} \in L^{q}, 1 \leqq p, q \leqq \infty, p^{-1}+q^{-1}=1$.

Recall that a function $f:[0,1] \rightarrow \mathbb{R}$ is of bounded variation if $\operatorname{var}(f)=\sup \left\{\sum_{i=1}^{n}\left|f\left(x_{i}\right)-f\left(x_{i-1}\right)\right|: 0 \leqq x_{0}<\ldots<x_{n} \leqq 1\right\}<\infty$,

and that

$$
B V=\left\{f \in L^{1} \mid \exists \text { a version } \tilde{f} \text { of } f \text { with } \operatorname{var}(\tilde{f})<\infty\right\}
$$

is a linear subspace of $L^{1}$. On $B V$ one can define a norm

$$
\|f\|_{v}:=\max \left\{\|f\|_{1}, \inf \{\operatorname{var}(\tilde{f}) \mid \tilde{f} \text { a version of } f\}\right\} .
$$

$\left(B V,\|\cdot\|_{v}\right)$ is a Banach-space and $\left\{f \in B V:\|f\|_{v} \leqq 1\right\}$ is a compact subset of $L^{1}$.

In $[4,13]$ it has been shown that if $g:[0,1] \rightarrow(0, \infty)$ is of bounded variation and if $\vartheta<1$, then $P_{\mid B V}: B V \rightarrow B V$ is a quasi-compact operator, i.e. $P$ has only a finite number of eigenvalues $1=\lambda_{1}, \ldots, \lambda_{r}$ of modulus 1 , whose corresponding eigenspaces are finite-dimensional. $P^{n}$ can be written as

$$
P^{n}=\sum_{i=1}^{r} \lambda_{i}^{n} \Phi_{i}+\Psi^{n}, \quad(n \in \mathbb{N})
$$

where the $\Phi_{i}$ are eigenprojections and $\left\|\Psi^{n}\right\|_{v} \leqq M q^{n}$ for some $M>0,0<q<1$. The function $h:=\Phi_{1}(1)$ is a $P$-invariant probability density such that for every $f \in L^{\infty}$ holds

$$
\int f(T x) \cdot h(x) d x=\int f(x) P h(x) d x=\int f(x) h(x) d x,
$$

i.e. $d \mu(x)=h(x) d x$ is a $T$-invariant probability on $[0,1]$. From (1) it follows that there is a power $T^{k}$ and a finite partition $\left\{A_{1}, \ldots, A_{s}\right\}$ of $[0,1]$ into $T^{k}$-invariant sets (each of them a finite union of intervals, see [6]) such that $T_{\mid A_{i}}^{k}$ is mixing $(i=1, \ldots, s)$. If $T$ itself is mixing (for sufficient conditions see e.g. [1]), (1) reduces to

$$
P^{n}=\Phi_{1}+\Psi^{n} \quad \text { and } \quad \Phi_{1} f=h \cdot \int f(x) d x .
$$

If $T$ is not mixing, we consider instead the $T_{\mid A_{i}}^{k}$, which is possible because the $A_{i}$ are finite unions of intervals. For mixing $T$ it follows from (2) that each initial density $f \in B V$ converges exponentially fast under the action of the system to the equilibrium density $h$ :

$$
\left\|P^{n} f-h\right\|_{v}=\left\|P^{n} f-\Phi_{1} f\right\|_{v}=\left\|\Psi^{n} f\right\|_{v} \leqq M \cdot q^{n} \cdot\|f\|_{v} .
$$

This can be expressed in terms of the spectrum $\sigma(P \mid B V)$ of $P: B V \rightarrow B V$ as

$$
\sigma(P \mid B V)=\sigma(\Psi \mid B V) \cup\{1\} \cong\left\{|z| \leqq q_{0}\right\} \cup\{1\},
$$


where $q_{0}$ is the infinum of all numbers $q$ that can occur in (3). In order to get more information about the approach of $P^{n} f$ to $h$ as $n \rightarrow \infty$ one should relate $q_{0}$ to other dynamical characteristics of the system and give a more detailed description of $\sigma(P \mid B V)$. We present here some partial results in this direction: In Sect. II we show that $q_{0} \geqq \vartheta$, more precisely: $\vartheta$ is the radius of the essential spectrum of $P_{\mid B V}$ (see [9]). Having decomposed the system into the wandering and the non-wandering part (modulo sets of Lebesgue-measure 0) in Sect. III, we discuss the spectrum of $P$ restricted to the non-wandering set in Sect. IV and show that for continuous $T$ each $\lambda \in \mathbb{C}$ with $|\lambda|<\exp (-h(T))$ is an eigenvalue of $T$. $[h(T)$ is the topological entropy of T.] A simple example is treated in detail in Sect. V. It suggests a connection between the metric entropy and the speed of convergence to equilibrium for Hölder-continuous densities, which is inspired by ideas of Parry and Tuncel [10]. In Sect. VI we finally discuss the transition from the wandering to the non-wandering set and relate the transition rate to the pressure of $T_{\mid \Sigma}$ on $\log g$, where $\Sigma$ is the set of those non-wandering points which do not belong to the support of the invariant measure $\mu$. This is related to results of Pianigiani/Yorke [12] and Pianigiani [11] on exponential decay.

\section{The Essential Spectrum}

Following Browder [2], Definition 11, we say that a complex number $\lambda$ belongs to the essential spectrum ess $(P \mid B V)$ of $P_{\mid B V}$, whenever at least one of the following conditions holds:

i) The range of $(T-\lambda I)$ is not closed in $B V$.

ii) $\bigcup_{n \geq 0} \operatorname{ker}\left((T-\lambda I)^{n}\right)$ is of infinite dimension.

iii) $\lambda$ is a limit point of $\sigma(P \mid B V)$.

The set ess $(P \mid B V)$ is closed, and $\sigma(P \mid B V) \backslash \operatorname{ess}(P \mid B V)$ consists of at most countably many isolated points which have no limit points outside ess $(P \mid B V)$. For each $\lambda \in \sigma(P \mid B V) \backslash \operatorname{ess}(P \mid B V)$ the spectral-projection associated to $\lambda$ has finitedimensional range since

$$
\operatorname{dim}\left(\bigcup_{n \geqq 0} \operatorname{ker}\left((T-\lambda I)^{n}\right)<\infty\right) .
$$

Define $r_{e}=\sup \{|\lambda|: \lambda \in \operatorname{ess}(P \mid B V)\}$ the radius of the essential spectrum of $P_{\mid B V}$. Nussbaum [9] has shown that

$$
r_{e}=\lim _{n \rightarrow \infty}\left(\left\|P^{n}\right\|_{K}\right)^{1 / n},
$$

where $\left\|P^{n}\right\|_{K}=\inf \left\{\left\|P^{n}-K\right\|_{v}: K\right.$ is a compact linear operator on $\left.B V\right\}$. (The " $\leqq$ "direction of (5) follows immediately from Lemma VIII.8.2 in [3].) We show (without assuming $T$ is mixing):

1. Theorem. $r_{e}=\lim _{n \rightarrow \infty}\left(\left\|g_{n}\right\|_{\infty}\right)^{1 / n}=\vartheta$.

Proof. In Proposition 2 of [13] it has been shown that for sufficiently big $n \in \mathbb{N}$ there exists a finite-dimensional operator $K$ with $\left\|P^{n}-K\right\|_{v}<1$. A closer look at the proof reveals that even more has been shown: $K$ can be chosen such that $\left\|P^{n}-K\right\|_{v} \leqq 6 \cdot \sup \left\{g_{n}(x)\right\}$. Now $r_{e} \leqq \vartheta$ follows from (5). For $f:[0,1] \rightarrow \mathbb{C}$ of 

bounded variation call $f\left(x^{+}\right)=\lim _{y \downarrow x} f(y)$ and $f\left(x^{-}\right)=\lim _{y \uparrow x} f(y)$. We show now that
for $n \in \mathbb{N}$ and $x \in[0,1]$,

$$
\left\|P^{n}\right\|_{K} \geqq\left|\left(T^{n}\right)^{\prime}\left(x^{ \pm}\right)\right|^{-1} \text {. }
$$

For $\varepsilon>0$ there is $\delta>0$ such that $\left.T^{n}\right|_{(x, x+\delta]}$ is monotone and differentiable and $\left.||\left(T^{n}\right)^{\prime}\left(x^{+}\right)\right|^{-1}-\left|\left(T^{n}\right)^{\prime}(y)\right|^{-1} \mid<\varepsilon$ for $x<y \leqq x+\delta$. (Assume $x<1$ here.) Consider functions $f_{k}:=1_{\left(c_{k}, c_{k-1}\right]}$ with $c_{k}=x+2^{-k} \delta(k=1,2,3, \ldots)$. Then

$$
\begin{gathered}
\left\|f_{k}\right\|_{v}=2, \quad\left\|f_{k}-f_{l}\right\|_{v}=4 \quad \text { for } k \neq l, \\
\left\|P^{n} f_{k}\right\|_{v}=\left\|1_{\left(T^{n} c_{k}, T^{n} c_{c_{k}-1}\right]} \cdot \frac{1}{\left|\left(T^{n}\right)^{\prime}\right| \circ T^{-n}}\right\| v \\
\geqq 2\left(\left|\left(T^{n}\right)^{\prime}\left(x^{+}\right)\right|^{-1}-\varepsilon\right)=: \alpha, \\
\left\|P^{n} f_{k}-P^{n} f_{l}\right\|_{v}=\left\|P^{n} f_{k}\right\|_{v}+\left\|P^{n} f_{l}\right\|_{v} \geqq 2 \alpha \text { for } k \neq l .
\end{gathered}
$$

Let $K: B V \rightarrow B V$ be a compact linear operator and denote by $C$ the closure of $\left\{K f_{k}: k \in \mathbb{N}\right\} . C$ is compact. Hence for each $k$ there is $\varrho_{k} \in C$ such that $\left\|P^{n} f_{k}-\varrho_{k}\right\|_{v}$ $=\inf \left\{\left\|P^{n} f_{k}-\varrho\right\|_{v}: \varrho \in C\right\}$, and there is a subsequence $\varrho_{k_{v}}$ converging to some $\varrho \in C$.

Now

$$
\left\|P^{n} f_{k_{v}}-K f_{k_{v}}\right\|_{v} \geqq\left\|P^{n} f_{k_{v}}-\varrho_{k_{v}}\right\|_{v} \geqq\left\|P^{n} f_{k_{v}}-\varrho\right\|_{v}-\varepsilon
$$

for $v$ big enough. On the other hand we have because of (9):

$$
\left\|P^{n} f_{k}-\varrho\right\|_{v} \geqq \alpha \quad \text { or } \quad\left\|P^{n} f_{l}-\varrho\right\|_{v} \geqq \alpha \quad \text { if } \quad k \neq l .
$$

Hence there are infinitely many $k$ for which $\left\|\left(P^{n}-K\right) f_{k}\right\|_{v} \geqq \alpha-\varepsilon$. As $\varepsilon>0$ was arbitrary this implies (6) for $x^{+}$, and the same argument applies to the $x^{-}$-part, too. As $\left|\left(T^{n}\right)^{\prime}\right|^{-1}=g_{n}$ is of bounded variation, the theorem follows from (6).

\section{The Decomposition}

Set $S=\operatorname{supp}(\mu) . S$ is a finite union of intervals (cf. [6]). As $T S \subseteq \operatorname{closure}(S)$, we have

$$
P^{n} f=P^{n}\left(f \cdot 1_{S}\right)+P^{n}\left(f \cdot 1_{S^{c}}\right)=P_{S}^{n} f_{\mid S}+P^{n}\left(f \cdot 1_{S^{c}}\right),
$$

where $P_{S}$ is the Perron-Frobenius-operator associated to $T_{\mid S}$ and acting on the space of integrable functions on $S$. As $S$ is a finite union of intervals, all results on piecewise monotonic transformations on $[0,1]$ apply to $T_{\mid S}$. Therefore we can treat the following two problems separately:

a) The behaviour of $P^{n} f$, if $S=[0,1]$.

b) The behaviour of $P^{n}\left(f \cdot 1_{S^{c}}\right)$ on $S^{c}$, i.e., the transition from $S^{c}$ to $S$.

\section{Eigenvalues and Eigenfunctions}

In this section we neglect transition phenomena from the wandering to the nonwandering set and assume $S=[0,1]$. Thus $T$ is onto, and if we choose a lower semi-continuous version of $h$, then $h(x)>0$ on an open set of full measure. As $T$ leaves $\mu$ invariant, it acts isometrically on each $L_{\mu}^{p}(1 \leqq p \leqq \infty)$, and we can define 
$T^{*}: L_{\mu}^{2} \rightarrow L_{\mu}^{2}$ by $\left\langle T^{*} f_{1}, f_{2}\right\rangle=\left\langle f_{1}, T f_{2}\right\rangle$. Most of the following simple facts can be found in [4], Lemmas 8, 9 and Theorem 3:

$$
\begin{gathered}
T^{*} f=\frac{P(f \cdot h)}{h} \text { and consequently } T^{*} \text { extends to } L_{\mu}^{1},\left\|T^{*}\right\|_{1}=1, \\
\left|T^{*} f\right| \leqq\|f\|_{\infty} \cdot \frac{P h}{h}=\|f\|_{\infty}, \text { i.e. }\left\|T^{*}\right\|_{\infty}=1, \\
\left(T^{*}\right)^{n} T^{n}=\mathrm{Id} \text { for all } n \in \mathbb{N},
\end{gathered}
$$

$T^{n}\left(T^{*}\right)^{n}$ is the orthogonal projection onto $T^{n} L_{\mu}^{2}$, and $\bigcap_{n=0}^{\infty} T^{n} L_{\mu}^{2}$

is finite-dimensional (this is less obvious).

For $\lambda \in \mathbb{C},|\lambda|<1$ define $Q_{\lambda}: L_{\mu}^{1} \rightarrow L_{\mu}^{1}$ by

$$
Q_{\lambda}:=\sum_{k=0}^{\infty} \lambda^{k} T^{k}\left(\mathrm{Id}-T T^{*}\right)
$$

One easily checks:

$$
\begin{gathered}
T^{*} Q_{\lambda}=\lambda Q_{\lambda}, \\
Q_{\lambda}^{2}=Q_{\lambda}, \\
Q_{\lambda} f=f \Leftrightarrow T^{*} f=\lambda f \Leftrightarrow P(f \cdot h)=\lambda(f \cdot h) \text { for } f \in L_{\mu}^{1} .
\end{gathered}
$$

Hence $Q_{\lambda}$ is a projection onto $\left\{f \in L^{1}: P(f h)=\lambda(f \cdot h)\right\}$ and $\lambda$ is a $L^{1}$-eigenvalue of $P$ if and only if $Q_{\lambda} \neq 0$. Set $Q_{\lambda}^{(n)}:=\sum_{k=0}^{n-1} \lambda^{k} T^{k}\left(\operatorname{Id}-T T^{*}\right)$.

2. Lemma. There is a function $\tilde{f} \in B V$ such that for all $n \in \mathbb{N}$ holds: $Q_{\lambda}^{(n)} \tilde{f} \neq Q_{\lambda}^{(n+1)} \tilde{f}$, $\left\|Q_{\lambda}^{(n)} \tilde{f}\right\|_{\infty}<\infty$. Furthermore $\tilde{f}$ can be chosen such that there is $\delta>0$ and $\tilde{f}(x)=0$ if $h(x)<\delta$.

Proof. As $\bigcap_{n=0}^{\infty} T^{n} L_{\mu}^{2}$ is finite-dimensional, $T L_{\mu}^{2} \neq L_{\mu}^{2}$. Hence, as $B V$ is dense in $L_{\mu}^{2}$, there is $f \in B V$ with $T T^{*} f \neq f$. As $\{h(x)>0\}$ is an open set of full measure, there is a sequence $U_{k} \subseteq[0,1]$ of finite unions of intervals such that $h_{\mid U_{k}} \geqq \frac{1}{k}$ and $m\left(U_{k}\right) \rightarrow 1$ $(k \rightarrow \infty)$. Set $f_{k}:=f \cdot 1_{T^{-1} U_{k}}$. Then $f_{k} \in B V, T T^{*} f_{k} \neq f_{k}$ for big $k$, and $f_{k}(x)=0$ if $h(x)<1 / k$. Take $\tilde{f}=f_{k}$ for some big $k$. If $Q_{\lambda}^{(n)} \tilde{f}=Q_{\lambda}^{(n+1)} \tilde{f}$, then $\lambda^{n} T^{n}\left(\tilde{f}-T T^{*} f\right)=0$, contradicting the fact that $T$ is onto. Finally, $\left\|Q^{(n)} \tilde{f}\right\|_{\infty}<\infty$ as $\left\|T^{*}\right\|_{\infty}=1$.

3. Proposition. For $\lambda \in \mathbb{C}$ with $|\lambda|<1$ there is at least one eigenfunction $0 \neq f \in L^{\infty}$ with $P f=\lambda f$. Hence

$$
\{|z|<1\} \cong \text { eigenvalues }\left(P \mid L^{p}\right) \leqq \sigma\left(P \mid L^{p}\right)=\{|z| \leqq 1\} \quad \text { for } 1 \leqq p \leqq \infty \text {. }
$$


Proof. Choose $n$ such that $\left|\lambda^{n}\right|<1 / 2$. Without loss we may assume by Lemma 2 that there is $\tilde{f} \in B V$ such that $Q_{\lambda}^{(n)} \tilde{f} \neq 0$. From $Q_{\lambda} \tilde{f}=Q_{\lambda}^{(n)} \tilde{f}+\sum_{k=1}^{\infty} \lambda^{k n} T^{k n} Q_{\lambda}^{(n)} \tilde{f}$ and

$$
\left.\left\|\sum_{k=1}^{\infty} \lambda^{k n} T^{k n} Q_{\lambda}^{(n)} \tilde{f}\right\|_{\infty}<\left\|Q_{\lambda}^{(n)} \tilde{f}\right\|_{\infty} \quad \text { (as }\left|\lambda^{n}\right|<\frac{1}{2}\right)
$$

if follows that $0<\left\|Q_{\lambda} \tilde{f}\right\|_{\infty}<\infty$, and $Q_{\lambda} \tilde{f}$ is the desired eigenfunction.

This result shows that the spectrum of $P$ as operator on $L^{p}$ contains no useful information. In some nice cases, however, one can conclude that the eigenfunction $f$ is of bounded variation from the above construction. For continuous $T$ it has been shown in $[8,17]$ that

$$
\lim _{n \rightarrow \infty} \frac{1}{n} \log \operatorname{card} \mathscr{I}_{n}=h(T),
$$

where $\mathscr{I}_{n}=\bigvee_{i=0}^{n-1} T^{-i} \mathscr{I}$ and $h(T)$ is the topological entropy of $T$. For piecewise continuous $T$ we may take this as a definition of $h(T)$.

4. Proposition. For $\lambda \in \mathbb{C}$ with $|\lambda|<\exp (-h(T))$ there is at least one eigenfunction $0 \neq f \in B V$ with $P f=\lambda f$. Hence

$$
\{|z|<\exp (-h(T))\} \cong \text { eigenvalues }(P \mid B V) .
$$

Proof. In the proof of the preceding proposition $0 \neq f$ had been constructed as $f=Q_{\lambda} \tilde{f}$ for some $\tilde{f} \in B V$ with $\tilde{f}(x)=0$ if $h(x)<\delta$. One easily sees that $c:=\left\|\tilde{f}-T T^{*} \tilde{f}\right\|_{v}<\infty$. Hence for $|\lambda|<\exp (-h(T))$ holds:

$$
\begin{aligned}
\|f\|_{v} & \leqq \sum_{k=0}^{\infty}|\lambda|^{k}\left\|T^{k}\left(\tilde{f}-T T^{*} \tilde{f}\right)\right\|_{v} \\
& \leqq \sum_{k=0}^{\infty}|\lambda|^{k} \sum_{I \in \mathscr{I}_{k}}\left(c+2 \cdot\left\|\tilde{f}-T T^{*} \tilde{f}\right\|_{\infty}\right)<\infty .
\end{aligned}
$$

Remarks. 1) If $\left|T^{\prime}\right|=\beta>1$ is constant, it follows from Theorem 1 and Proposition 4 that $\operatorname{ess}(P \mid B V)=\{|z| \leqq \beta\}$ and that the interior of $\operatorname{ess}(P \mid B V)$ consists of eigenvalues.

2) In some particularly nice cases, $\sigma(P \mid B V)=\operatorname{ess}(P \mid B V) \cup\{1\}$, and $r_{e}=\vartheta$ describes the approach of $P^{n} f$ to $h$ in $\|\cdot\|_{v}$-norm. One might hope to get better convergence rates, if one considers $\left\|P^{n} f-h\right\|_{1}$. That this is not the case will become clear in the following example.

\section{An Example}

For a compact subset $C$ of $L^{1}$ define $\gamma_{c}:=\limsup _{n \rightarrow \infty}\left(\sup \left\{\left\|\Psi^{n} f\right\|_{1}: f \in C\right\}\right)^{1 / n}$. We consider the following simple example:

Example. Fix $0<p, q<1$ with $p+q=1$ and define

$$
T_{p}(x)=p^{-1} x \text { if } 0 \leqq x \leqq p, \quad T_{p}(x)=q^{-1}(x-p), \quad \text { if } \quad p \leqq x \leqq 1 .
$$


Then $\exp (-h(T))=1 / 2$ for all $p, \vartheta\left(T_{p}\right)=\max (p, q)$, and one easily shows that $T$ is mixing (cf. [1], Theorem 2a) and leaves the Lebesgue-measure $m$ invariant. Furthermore $\sigma(P \mid B V)=\operatorname{ess}(P \mid B V) \cup\{1\}$, if $P$ is the Perron-Frobenius-operator of $T_{p}$. Set $C_{v}:=\left\{f \in B V:\|f\|_{v} \leqq 1\right\}$, and for $r>0: C_{r}:=\left\{f \in L^{1}:\|f\|_{1} \leqq 1\right.$ and a version $\tilde{f}$ of $f$ is Hölder-continuous with exponent $r$ and $\left.H_{r}(\tilde{f}) \leqq 1\right\}$, where

$$
H_{r}(\tilde{f})=\sup \left\{\frac{|\tilde{f}(x)-\tilde{f}(y)|}{|x-y|^{r}}: x, y \in[0,1]\right\} .
$$

a) As $\sigma(P \mid B V)=\operatorname{ess}(P \mid B V) \cup\{1\}$, we have $\gamma_{c_{v}}\left(T_{p}\right) \leqq r_{e}=\vartheta\left(T_{p}\right)=\max \{p, q\}$, and the following example shows that there is even equality (suppose $p \geqq q$ ): Set $a_{n}=\frac{1}{2} n^{-2}\left(\sum_{k=1}^{\infty} k^{-2}\right)^{-1}$ and $f:=\sum_{k=1}^{\infty} a_{k} 1_{\left[0, p^{k}\right]}$.

Then $\|f\|_{v}=1$ and $\left\|\Psi^{n} f\right\|_{1}=\sum_{k=n+1}^{\infty} a_{k} p^{k}$. Hence $\limsup _{n \rightarrow \infty}\left(\left\|\Psi^{n} f\right\|_{1}\right)^{1 / n}=p$.

b) Let $f \in C_{r}, f_{0}=f-\int f d m$. Then

$$
\begin{aligned}
\Psi^{n} f & =P^{n} f_{0}=\sum_{I \in \mathscr{A}_{n}} m(I)\left(f_{0} \circ T_{\mid I}^{-1}\right), \quad \text { such that } \\
H_{r}\left(\Psi^{n} f\right) & \leqq H_{r}(f) \cdot \sum_{I \in \mathscr{I}_{n}} m(I)\left(\sup _{I} g_{n}\right)^{r} \\
& =H_{r}(f) \cdot \sum_{I \in \mathscr{I}_{n}} m(I)^{1+r}=H_{r}(f)\left(p^{1+r}+q^{1+r}\right)^{n} .
\end{aligned}
$$

Hence $\gamma_{C_{r}}\left(T_{p}\right) \leqq p^{1+r}+q^{1+r}$, and equality holds because of the following example: Let $f(x)=2 x\left(0 \leqq x \leqq \frac{1}{2}\right), f(x)=2(1-x)\left(\frac{1}{2} \leqq x \leqq 1\right)$ and $f_{n}(x)=\frac{1}{2}\left(g_{n}(x)\right)^{r} f\left(T_{p}^{n} x\right)$. Then

$$
\begin{aligned}
H_{r}\left(f_{n}\right) & =1,\left\|f_{n}\right\|_{1} \leqq 1, \text { and } \\
\left\|\Psi^{n} f_{n}\right\|_{1} & =\left\|P^{n} f_{n}-\int f_{n} d m\right\|_{1}=\left\|\frac{1}{2} f \cdot P^{n} g_{n}^{r}-\frac{1}{4} \int g_{n}^{r} d m\right\|_{1} \\
& =\left\|\left(\frac{1}{2} f-\frac{1}{4}\right)\left(\sum_{I \in \mathscr{I}_{n}} m(I)^{1+r}\right)\right\|_{1} \text { as } g_{n}(I)=m(I) \\
& =\frac{1}{8}\left(p^{1+r}+q^{1+r}\right)^{n} .
\end{aligned}
$$

In particular, all coefficients $\gamma_{C_{r}}$ are different from $\exp \left(-h_{m}\left(T_{p}\right)\right)=p^{p} \cdot q^{q}$. If we extend the function $r \rightarrow \gamma_{C_{r}}(r>0)$ to $r=0$ by $\gamma_{C_{0}}=1$, then we see, however, that $\frac{d}{d r} \gamma_{c_{r \mid r=0}}=p \log p+q \log q=-h_{m}\left(T_{p}\right)$, i.e., $\gamma_{C_{r}}\left(T_{p}\right)=1-r \cdot h_{m}\left(T_{p}\right)+o(r)$. That there is some hope to generalize this relation may be seen from the following: for $T=T_{p}$ we have

$$
\begin{aligned}
\gamma_{C_{r}}(T) & =\lim _{n \rightarrow \infty}\left(\sum_{I \in \mathscr{G}_{n}} m(I)\left(g_{n}(I)\right)^{r}\right)^{1 / n}=\lim _{n \rightarrow \infty}\left(\int g_{n}^{r} d m\right)^{1 / n} \\
& =\lim _{n \rightarrow \infty}\left(\int P^{n} g_{n}^{r} d m\right)^{1 / n}=\lim _{n \rightarrow \infty}\left(\int P_{1+r}^{n}(1) d m\right)^{1 / n}
\end{aligned}
$$

where $P_{1+r} f=\sum_{I \in \mathscr{I}}\left(f \cdot g^{1+r}\right) \circ T_{\mid I}^{-1}$. 
Parry and Tuncel [10] have shown that in such a situation where $T=T_{p}$ is isomorphic to a Markov-shift, $P_{1+r}^{n}(1) \sim \lambda(r)^{n} \cdot h_{r}\left(h_{r}\right.$ is an eigenfunction of $P_{1+r}$ to the greatest positive eigenvalue $\lambda(r))$ and that $\frac{d}{d r} \lambda(r)_{\mid r=0}=-h_{m}\left(T_{p}\right)$. Hence we recover

$$
\frac{d}{d r} \gamma_{C_{r}}\left(T_{p}\right)_{\mid r=0}=-h_{m}\left(T_{p}\right)
$$

Using results on equilibrium states in [5] it might be possible to use similar arguments for non-Markovian transformations, too.

\section{The Transition}

Let $U \subseteq[0,1]$ be finite union of intervals, $A:=[0,1] \backslash U$. The transition (under $T)$ from $A$ to $U$ can be modeled by the transformation $\tilde{T}:[0,1] \rightarrow[0,1]$,

$$
\tilde{T}(x)=T(x) \quad \text { if } \quad x \in A, \quad \tilde{T}(x)=x \quad \text { if } \quad x \in U .
$$

$\widetilde{T}$ is again a piecewise monotonic transformation, and its associated PerronFrobenius-operator $\tilde{P}$ describes the extinction of $A$ (i.e., the transition from $A$ to $U$ ) in the following sense: Given an initial density $f$ on $A$ which defines a probability $d v(x)=f(x) d x$, for $B \subseteq A$ holds:

$$
\begin{aligned}
v\{x & \left.: T^{k} x \in A \quad(k=0, \ldots, n-1), T^{n} x \in B\right\} \\
& =v\left\{x: \widetilde{T}^{n} x \in B\right\} \\
& =\int_{B} \widetilde{P}^{n} f(x) d x,
\end{aligned}
$$

in particular:

$$
D_{f}(n):=v\left\{x: T^{k} x \in A(k=0, \ldots, n)\right\}=\int_{A} \widetilde{P}^{n} f(x) d x .
$$

If $U=S$ is the support of the absolutely continuous invariant measure (cf., Sect. III), (10) and (11) describe the transition from the wandering to the nonwandering set.

For our further investigations we need not assume that $\vartheta<1$. However, we suppose that there is $R>0$ such that $R^{-1} \leqq\left|\widetilde{T}^{\prime}(x)\right| \leqq R$ for all points $x$ where $T$ is differentiable and that $\widetilde{T}^{\prime}$ is of bounded variation. Our aim is to relate $D_{f}(n)$ to the pressure of $\tilde{T}$ under $-\log \left|\tilde{T}^{\prime}\right|$. Since the concept of pressure is defined for continuous transformations only, we first have to modify the space $[0,1]$ and its topology in such a way that $\widetilde{T}$ and $\widetilde{T}^{\prime}$ become continuous. The idea is due to Walters [15] and has been carried out for general piecewise monotonic transformations in $[4,5]$. Let $W_{0}$ be the at most countable set of discontinuities of $\widetilde{T}$ and $\tilde{T}^{\prime}$ and set $W=\bigcup_{j=0}^{\infty} T^{-j} W_{0} \backslash\{0,1\} . \tilde{T}$ and $\tilde{T}^{\prime}$ are continuous on

$$
[0,1] \backslash W, \quad \tilde{T}([0,1] \backslash W) \subseteq([0,1] \backslash W) \cup\{0,1\},
$$


and as $\tilde{T}, \tilde{T}^{\prime}$ are of bounded variation, one-sided limits of $\tilde{T}$ and $\tilde{T}^{\prime}$ exist in all points of $[0,1]$. Let $W^{\prime}=\left\{x^{\prime}: x \in W\right\}$ be a disjoint copy of $W$ and set $X=[0,1] \cup W^{\prime}$. We extend the order relation on $[0,1]$ to $X$. If $x<y<z$ in $[0,1]$ and $y \in W$, then we define $x<y<y^{\prime}<z$. The resulting order topology is compact and separable, $\widetilde{T}:[0,1] \backslash W \rightarrow X$ can be extended to a continuous transformation on $X$ and also $\widetilde{T}^{\prime}:[0,1] \backslash W \rightarrow \mathbb{R}$ can be continuously extended, just as any other $f \in B V$. The Lebesgue-measure carrys over to $X$. Let $\tilde{\mathscr{I}}$ be the partition of $[0,1]$ into intervals on which $\tilde{T}$ is monotone and continuous, $\tilde{\mathscr{I}}_{n}=\bigvee_{i=0}^{n-1} \widetilde{T}^{-i} \tilde{\mathscr{F}}$. Denote by $\overline{\mathscr{I}}_{n}$ the corresponding partition of $X$ into closed intervals: $\overline{\mathscr{I}}_{n}=\left\{\bar{I} \cong X: I \in \widetilde{\mathscr{I}}_{n}\right\}$, where $\bar{I}$ is the closure of $I$ in $X$. ( $(\bar{I} \cap \bar{J}=\emptyset$ if $I \cap J=\emptyset$ by construction of $X$.) As $X$ is separable it is metrizable by a metric $d(\cdot, \cdot)$, and

$$
\delta:=\min \{\operatorname{dist}(\bar{I}, \bar{J}): I, J \in \mathscr{I}, I \cap J=\emptyset\}>0 .
$$

Suppose that $x, y, z \in X, \widetilde{T}^{n} y=\widetilde{T}^{n} z=x, y \neq z$. Let $k=\min \left\{i \geqq 0 \mid \widetilde{T}^{i} y=\tilde{T}^{i} z\right\}$. Then $1 \leqq k \leqq n$ and $\widetilde{T}^{k-1} y \neq \widetilde{T}^{k-1} z$. If there were $\bar{I}$ in $\bar{I}$ such that $\widetilde{T}^{k-1} y, \widetilde{T}^{k-1} z \in \bar{I}$, it would follow that $\widetilde{T}^{k} y \neq \widetilde{T}^{k} z$ as $\tilde{T}$ is injective on $\bar{I}$, contradicting the definition of $k$. Hence $d\left(\widetilde{T}^{k} y, \widetilde{T}^{k} z\right) \geqq \delta$. Therefore for each $x \in X$ holds: $\widetilde{T}^{-n}\{x\}$ is $(n, \delta / 2)$-separated (see [15]).

Consequently, if $0 \leqq f: X \rightarrow \mathbb{R}$ is bounded and $\tilde{g}: X \rightarrow \mathbb{R}$ is continuous with $R^{-1} \leqq \tilde{g}(x) \leqq R(x \in X)$ and $\tilde{g}(x)=1 /\left|T^{\prime}(x)\right|=1 /\left|\widetilde{T}^{\prime}(x)\right|(x \in A)$, one has with $\tilde{g}_{n}=\tilde{g}$ $\cdot \tilde{g} \circ \stackrel{\widetilde{T}}{\bar{T}} \cdot \ldots \cdot \tilde{g} \circ \widetilde{T}^{n-1}$ :

$$
\begin{aligned}
\limsup _{n \rightarrow \infty} n^{-1} \log D_{f}(n) & =\limsup _{n \rightarrow \infty} n^{-1} \log \int_{A} \sum_{y \in \widetilde{T}^{-} n_{x}}\left(f \cdot \tilde{g}_{n}\right)(y) d x \\
& \leqq \limsup _{n \rightarrow \infty} n^{-1} \log \left(\sup _{x \in A} \sum_{y \in \tilde{T}^{-n} n_{x}} \tilde{g}_{n}(y)\right) \\
& \leqq \operatorname{Pr}(\widetilde{T}, \log \tilde{g}),
\end{aligned}
$$

where $\operatorname{Pr}(\widetilde{T}, \log \tilde{g})$ denotes the pressure of $\widetilde{T}$ with respect to the energy-function $\log \tilde{g}$ (see [16]). This proves one half of the following theorem. Let $\Sigma=\bigcap_{n=0}^{\infty} \widetilde{T}^{-n} A$, $\tilde{g}$ as above.

5. Theorem. Suppose that $\sup _{U} \tilde{g} \leqq \inf _{A} \tilde{g}$, and that there is $M>0$ such that $M^{-1} \leqq f(x) \leqq M(x \in A)$. If $h(\widetilde{T})>0$ or if $h(\widetilde{T})=0$ and $\inf \left\{m\left(A \cap \widetilde{T}^{n} X\right): n \in \mathbb{N}\right\}>0$, then

$$
\begin{aligned}
& \operatorname{Pr}(\tilde{T}, \log \tilde{g})+\sup _{n} n^{-1} \log \frac{\inf _{\Sigma}\left|\left(T^{n}\right)^{\prime}\right|}{\sup _{\Sigma}\left|\left(T^{n}\right)^{\prime}\right|} \\
& \quad \leqq \liminf _{n \rightarrow \infty} n^{-1} \log D_{f}(n) \leqq \limsup _{n \rightarrow \infty} n^{-1} \log D_{f}(n) \\
& \quad \leqq \operatorname{Pr}(\widetilde{T}, \log \tilde{g}) .
\end{aligned}
$$

Furthermore $\operatorname{Pr}(\widetilde{T}, \log \tilde{g})=\operatorname{Pr}\left(T_{\mid \Sigma},-\log \left|T^{\prime}\right|_{\mid \Sigma}\right)$. 
Corollary. If $\left|T^{\prime}\right|=\beta>1$ is constant, then

$$
\lim _{n \rightarrow \infty} n^{-1} \log D_{f}(n)=\operatorname{Pr}(\tilde{T},-\log \beta)=h(\tilde{T})-\log \beta=h(\tilde{T})-h(T),
$$

where $h(T), h(\widetilde{T})$ denote the topological entropies of $T$ and $\tilde{T}$.

Proof of the Corollary. Apply the theorem with $\tilde{g}=\beta^{-1}$. The second equality follows from Theorem 2.1 in [16] and the third one e.g. from Theorem 6 in [4] together with Theorem 1 in [7]. For continuous $T$ one can also use Proposition 2.2 in [17].

Proof of the Theorem. In view of (12) only the first inequality must be proved. Suppose $h(\widetilde{T})>0$. It follows from Theorem 1 in [5] that there is a constant $c>0$ and a finite union of intervals $F$ with $m(F \cap A)>0$ ( $m$ Lebesgue-measure), such that for all $x \in F$ and $n \in \mathbb{N}$ holds

$$
\operatorname{card} \tilde{T}^{-n}\{x\} \geqq c \cdot \exp (n \cdot h(\tilde{T})),
$$

and (11) implies

$$
\begin{aligned}
D_{f}(n) & \geqq M^{-1} \cdot \int_{A \cap F} \sum_{y \in T^{-n_{x}}} \tilde{g}_{n}(y) d x \\
& \geqq c \cdot M^{-1} \cdot m(F \cap A) \cdot \exp \left(n \cdot h(\tilde{T})+i_{n}\left(\tilde{T}^{-n} A\right)\right),
\end{aligned}
$$

where $i_{n}(B):=\log \left(\inf \left\{\tilde{g}_{n}(y): y \in B\right\}\right)$ for $B \subseteq X$. Similarly,

$$
s_{n}(B):=\log \left(\sup \left\{\tilde{g}_{n}(y): y \in B\right\}\right) .
$$

Set $\Omega=\Sigma \cup U . \Omega$ contains the non-wandering set of $\tilde{T}^{k}$ for all $k \in \mathbb{N}$ and $\tilde{T} \Omega \subseteq \Omega$. From Theorems 2.1, 2.2, and 4.12 in [16] it follows that

$$
\begin{aligned}
n \cdot h(\tilde{T}) & =n \cdot h\left(\tilde{T}_{\mid \Omega}\right)=h\left(\tilde{T}_{\mid \Omega}^{n}\right) \\
& \geqq \operatorname{Pr}\left(\tilde{T}_{\mid \Omega}^{n}, \log \tilde{g}_{n \mid \Omega}\right)-s_{n}(\Omega) \\
& =n \cdot \operatorname{Pr}(\tilde{T}, \log \tilde{g})-s_{n}(\Sigma),
\end{aligned}
$$

because $\sup _{U} \tilde{g} \leqq \inf _{A} \tilde{g}$. Together with (13) this leads to

$$
\begin{aligned}
& \liminf _{n \rightarrow \infty} n^{-1} \log D_{f}(n) \\
& \quad \geqq \operatorname{Pr}(\widetilde{T}, \log \tilde{g})+\liminf _{n \rightarrow \infty} n^{-1} i_{n}\left(\widetilde{T}^{-n} A\right)-\limsup _{n \rightarrow \infty} n^{-1} S_{n}(\Sigma) .
\end{aligned}
$$

Fix $k \in \mathbb{N}$ and set $n=k m+j, 0 \leqq j<k$. Then

$$
\begin{aligned}
i_{n}\left(\tilde{T}^{-n} A\right) & \geqq i_{j}\left(\tilde{T}^{-n} A\right)+\sum_{l=1}^{m} i_{k}\left(\tilde{T}^{-k l} A\right) \\
& \geqq j \cdot i_{1}(A)+m \cdot i_{k}(\Sigma)+\sum_{l=1}^{m}\left(i_{k}\left(\tilde{T}^{-k l} A\right)-i_{k}(\Sigma)\right) .
\end{aligned}
$$


As $\Sigma=\bigcap_{n=0}^{\infty} \tilde{T}^{-n} A,\left(i_{k}\left(\tilde{T}^{-k l} A\right)-i_{k}(\Sigma)\right) \rightarrow 0$ as $l \rightarrow \infty$ for fixed $k$. Hence

$$
\liminf _{n \rightarrow \infty} n^{-1} i_{n}(\Sigma) \geqq \liminf _{n \rightarrow \infty} n^{-1} i_{n}\left(\widetilde{T}^{-n} A\right) \geqq \liminf _{m \rightarrow \infty} \frac{m}{k m+j} i_{k}(\Sigma)=k^{-1} i_{k}(\Sigma),
$$

such that

$$
\liminf _{n \rightarrow \infty} n^{-1} i_{n}\left(\tilde{T}^{-n} A\right)=\sup _{n} n^{-1} i_{n}(\Sigma)=\lim _{n \rightarrow \infty} n^{-1} i_{n}(\Sigma) .
$$

By the subadditivity of the sequence $s_{n}(\Sigma)$, we have

$$
\limsup _{n \rightarrow \infty} n^{-1} S_{n}(\Sigma)=\inf _{n} n^{-1} S_{n}(\Sigma)=\lim _{n \rightarrow \infty} n^{-1} S_{n}(\Sigma) \text {. }
$$

Now the first inequality of the theorem follows from (14). If $h(\tilde{T})=0$ and $d:=\inf \left\{m\left(A \cap \widetilde{T}^{n} X\right): n \in \mathbb{N}\right\}>0$, then - cf. (11) -

$$
D_{f}(n) \geqq d \cdot \exp \left(i_{n}\left(\tilde{T}^{-n} A\right)\right)=d \cdot \exp \left(n \cdot h(\tilde{T})+i_{n}\left(\tilde{T}^{-n} A\right)\right),
$$

and the same proof as above works, because we needed $h(\tilde{T})>0$ only in order to establish (13).

As mentioned above,

$$
\operatorname{Pr}(\tilde{T}, \log \tilde{g})=\operatorname{Pr}\left(\widetilde{T}_{\mid \Omega}, \log \tilde{g}_{\mid \Omega}\right)=\max \left\{\operatorname{Pr}\left(\widetilde{T}_{\mid \Sigma}, \log \tilde{g}_{\mid \Sigma}\right), \operatorname{Pr}\left(\widetilde{T}_{\mid U}, \log \tilde{g}_{\mid U}\right)\right\}
$$

(see Theorem 2.2 in [16]). As $\widetilde{T}_{\mid U}=\mathrm{id}_{U}$, it follows that

$$
\operatorname{Pr}\left(\widetilde{T}_{\mid U}, \log \tilde{g}_{\mid U}\right)=\sup _{U} \log \tilde{g} \leqq \inf _{A} \log \tilde{g} \leqq \inf _{\Sigma} \log \tilde{g} \leqq \operatorname{Pr}\left(\tilde{T}_{\mid \Sigma}, \log \tilde{g}_{\mid \Sigma}\right),
$$

hence $\operatorname{Pr}(\tilde{T}, \log \tilde{g})=\operatorname{Pr}\left(\widetilde{T}_{\mid \Sigma},-\left.\log T^{\prime}\right|_{\mid \Sigma}\right)$. This completes the proof of the theorem. Remark. If there is a periodic source in $A$, then $\inf \left\{m\left(A \cap \tilde{T}^{n} X\right): n \in \mathbb{N}\right\}>0$.

In the proof of the theorem we already used the fact that the dynamics of $\tilde{T}$ are essentially determined by those of $T_{\mid \Sigma}$, namely:

a) The non-wandering set of $\tilde{T}$ is contained in $\Sigma \cup U$ and $\widetilde{T}_{\mid U}=\mathrm{id}_{U}$.

b) As a consequence of a), each invariant ergodic measure of $\tilde{T}$ is either a point mass on some $x_{0} \in U$ or is concentrated on $\Sigma$ and invariant under $T_{\mid \Sigma}$.

So it is natural to conjecture that for $x \in \widetilde{T}^{-n} A, n$ big, the trajectory of $x$ is for a long time very similar to a typical trajectory in $\Sigma$. If $\tilde{T}$ is a Markov-transformation with Markov-partition $\overline{\mathscr{I}}$ and if $1<\left|\widetilde{T}^{\prime}\right|$ is continuous on each element of $\overline{\mathscr{I}}$, it is not hard to make this precise: By choice of the topology on $X,\left(T_{\mid \Sigma}, \Sigma\right)$ is homeomorphic to a subshift of finite type over the set of symbols $\mathscr{S}=\left\{\bar{I} \in \bar{I}: \widetilde{T}^{k} \bar{I}\right.$ $\cap \bar{A} \neq \emptyset$ for all $k \geqq 0\}$. For simplicity suppose that $T_{\mid \Sigma}$ is mixing, i.e., that the $0-1$ matrix $A$ defining the corresponding subshift satisfies $A^{K}>0$ for some $K \in \mathbb{N}$. Tuncel [14] has shown that under these assumptions

$$
\operatorname{Pr}\left(T_{\mid \Sigma}, \log \tilde{g}_{\mid \Sigma}\right)=\lim _{n \rightarrow \infty} n^{-1} \log \left(\sum_{y \in T_{\mid \Sigma}^{-} n_{x}} f(y) \cdot \tilde{g}_{n}(y)\right)
$$

for all $x \in \Sigma$, if $M^{-1} \leqq f \leqq M$. As $T_{\mid \Sigma}^{-n}\{x\}=T^{-n}\{x\}$ for $x \in \Sigma$, this implies

$$
\operatorname{Pr}(\tilde{T}, \log \tilde{g})=\lim _{n \rightarrow \infty} n^{-1} \log \tilde{P}^{n} f(x) \text { for } \quad x \in \Sigma .
$$


Denote $\mathscr{S}_{n}:=\bigvee_{i=0}^{n-1} \tilde{T}^{-n} \mathscr{S}$. Fix $\bar{I} \in \mathscr{S}$. Then for each $x \in \bar{I}$ and $S \in \mathscr{S}_{n}$ with $S \subseteq \widetilde{T}^{-n} \mid \bar{I}$, $S \cap \widetilde{T}^{-n}\{x\}$ contains exactly one element. This property defines in a natural way a $1-1$-correspondence between the sets $\widetilde{T}^{-n}\{x\}$ and $\widetilde{T}^{-n}\{y\}$ if $x, y$ are elements of the same $\bar{I} \in \mathscr{S}$, and it is not hard to see that by continuity of $\tilde{g}_{\mid A}=1 /\left|T^{\prime}\right|_{\mid A}$ the relation $(15)$ carries over to all $x \in \bar{I}, \bar{I} \in \mathscr{S}$. In particular, $\lim _{n \rightarrow \infty} n^{-1} \log D_{f}(n)$ $=\operatorname{Pr}(\widetilde{T}, \log \tilde{g})$.

The papers of Pianigiani-Yorke [12] and Pianigiani [11] on exponential decay deal also with such Markovian situations. They give detailed results on the asymptotic behaviour of $\widetilde{P}^{n} f$, and treat $n$-dimensional transformations. However, they do not relate the decay-exponent to the pressure of $T_{\mid \Sigma}$.

In [5] one finds results on equilibrium states for piecewise monotonic transformations which yield a relation like (15) also for many non-Markovian transformations. But it seems very difficult in general to extend (15) to a set of points of positive Lebesgue-measure.

\section{Concluding Remark}

In Sects. V and VI we used generalized Perron-Frobenius-operators $\left(P_{1+r}\right.$ in Sect. V, $\tilde{P}$ in Sect. VI) to describe certain aspects of the time-evolution of absolutely continuous initial distributions although, in general, $P_{1+r}$ and $\tilde{P}$ have singular invariant measures (equilibrium states) which are supposed to be not directly observable. It would be interesting to know if also properties of these singular measures themselves are reflected by the time-evolution of certain initial densities.

\section{References}

1. Bowen, R.: Bernoulli maps of an interval. Israel J. Math. 28, 298-314 (1978)

2. Browder, F.E.: On the spectral theory of elliptic differential operators. I. Math. Ann. 142, $22-$ 130 (1961)

3. Dunford, N., Schwartz, J.T.: Linear operators, Part I. New York: Interscience 1957

4. Hofbauer, F., Keller, G.: Ergodic properties of invariant measures for piecewise monotonic transformations. Math. Z. 180, 119-140 (1982)

5. Hofbauer, F., Keller, G.: Equilibrium states for piecewise monotonic transformations. Ergod. Theor. Dynam. Syst. 2, 23-43 (1982)

6. Kowalski, Z.S.: Piecewise monotonic transformations and their invariant measures. Bull. Acad. Pol. Sci. 27, 63-69 (1979)

7. Ledrappier, F.: Principe variationnel et système dynamiques symboliques. Z. Wahrscheinlichkeitstheorie Verw. Gebiete 30, 185-202 (1974)

8. Misiurewicz, M., Szlenk, W.: Entropy of piecewise monotone mappings. Studia Math. 67, 45$63(1980)$

9. Nussbaum, R.D.: The radius of the essential spectrum. Duke Math. J. 37, 473-478 (1970)

10. Parry, W., Tuncel, S.: On the classification of Markov chains by finite equivalence. Ergod. Theor. Dynam. Syst. 1, 303-335 (1981)

11. Pianigiani, G.: Conditionally invariant measures and exponential decay. J. Math. Anal. Appl. 82, 75-88 (1981) 
12. Pianigiani, G., Yorke, J.A.: Expanding maps on sets which are almost invariant: decay and chaos. Trans. Am. Math. Soc. 252, 351-366 (1979)

13. Rychlik, M.: Bounded variation and invariant measures. Studia Math. LXXVI, 69-80 (1983)

14. Tuncel, S.: Conditional pressure and coding. Israel J. Math. 39, 101-112 (1981)

15. Walters, P.: Equilibrium states for $\beta$-transformations and related transformations. Math. $Z$. 159, 65-88 (1978)

16. Walters, P.: A variational principle for the pressure of continuous transformations. Am. J. Math. 97, 937-971 (1973)

17. Young, L.-S.: On the prevalence of horeshoes. Trans. Am. Math. Soc. 263, 75-88 (1981)

Communicated by O. E. Lanford

Received June 1983; in revised form May 9, 1984

Note added in proof. In a joint paper of F. Hofbauer and the author (to appear in J. Reine Angew. Math. 1984) it has been shown for piecewise linear interval maps $T$ that the ArtinMazur-Ruelle zeta-function of $T$ is meromorphic in $\left\{|z|<r_{e}^{-1}\right\}$ and that $\lambda^{-1}$ is a pole of the zetafunction if and only if $\lambda \in \sigma(P \mid B V) \backslash \operatorname{ess}(P \mid B V)$ (cf. Sect. II). 
